\title{
白肋烟分子标记遗传图谱的构建及部分性状的遗传剖析
}

\author{
蔡长春 $^{1}$ 柴利广 ${ }^{2}$ 王 毅 ${ }^{1}$ 徐芳森 $^{2}$ 张俊杰 $^{1} \quad$ 林国平 $^{1, *}$ \\ ${ }^{1}$ 中国烟草白胁烟试验站 / 湖北省烟草科研所, 湖北武汉 $430030 ;{ }^{2}$ 华中农业大学作物遗传改良国家重点实验室, 湖北武汉 430070
}

摘 要: 用 112 个 AFLP、 6 个 SRAP 标记构建了白胁烟的分子标记遗传连锁图谱, 包括 22 个连锁群(A1 A22), 总遗 传长度为 $1953.6 \mathrm{cM}$, 标记的平均间距为 $20.5 \mathrm{cM}$, 有 25 个标记表现偏分离 $(17.0 \%)$, 主要集中在第 $1 、 11$ 和 14 连锁 群上; 利用 Windows QTL Cartographer Ver. 2.5 软件进行 QTL 分析, 结果表明, 共检测到 11 个主效 QTL, 其中 7 个与 化学成分性状相关, 另外 4 个与农艺性状相关。与烟碱、总氮和总糖相关的 QTL 各 2 个(btnic1 和 btnic2)、2 个(bttn1 和 $b t t n 2)$ 和 3 个 $(b t t s 1 、 b t t s 2$ 和 $b t t s 3)$ ，与株高 $(b t p h) 、$ 茎围 $(b t l s)$ 、节距 $(b t p t)$ 和中部叶长 $(b t l)$ 相关的 QTL 各 1 个, 没有 检测到与总钾、总叶数和中部叶宽相关的 QTL，其中与烟碱和总氮相关的 QTL 处于共分离状态(分别为 btnic1 和 bttn 1), 表明烟叶烟碱合成与氮素代谢之间可能存在某种未知的生物学相关性, 11 个主效 QTL 对各性状表型变异的贡 献率在 $12.3 \% \sim 26.4 \%$ 之间。

关键词: 白胁烟; 遗传连锁图谱; 农艺性状; 遗传剖析

\section{Construction of Genetic Linkage Map of Burley Tobacco (Nicotiana tabacum L.) and Genetic Dissection of Partial Traits}

\author{
CAI Chang-Chun ${ }^{1}$, CHAI Li-Guang ${ }^{2}$, WANG Yi ${ }^{1}$, XU Fang-Sen ${ }^{2}$, ZHANG Jun-Jie ${ }^{1}$, and LIN Guo-Ping ${ }^{1, *}$ \\ ${ }^{1}$ Burley Tobacco Experimental Station of China, Hubei Tobacco Research Institute, Wuhan 430030, China; ${ }^{2}$ National Key Laboratory of Crop \\ Genetic Improvement, Huazhong Agricultural University, Wuhan 430070, China
}

\begin{abstract}
The agronomic traits and chemical components are very important factors affecting yield and tobacco leaf quality. Dissecting genetic control of these traits can facilitate the breeding of new tobacco cultivars by marker-assisted selection (MAS). The objective of this study was to identify QTLs controlling partial agronomic traits and chemical components in burley tobacco. A double haploid (DH) population of burley tobacco was used to construct a molecular marker genetic linkage map. The DH population was derived from a cross between high quality cultivar Burley 37 with high nicotine content and Burley 21 with low nicotine content. The mapping population was planted in main production region of burley tobacco in Hubei province for two years to obtain repetitive phenotype data. On the basis of this map, QTLs for four chemical components including nicotine (NIC), total nitrogen (TN), total sugar (TS), total potassium (TK) of air-cured central tobacco leaf and six agronomic traits including plant height (PH), stalk circumference (LS), distance between nodes (PT), number of total leaf (LN), length of central leaf (L) and width of central leaf (W) were analyzed by using software Windows QTL Cartographer Ver. 2.5. The results showed that a total of 112 AFLP loci and six SRAP loci assembled into 22 linkage groups (A1-A22) composed the whole linkage map spanning 1953.6 $\mathrm{cM}$ with an average distance of $20.5 \mathrm{cM}$ between adjacent loci. There were 25 distortion-segregation loci (17.0\%) mainly clustering in linkage groups A1, A11, and A14. A total of eleven main QTLs including seven QTLs influencing chemical components and the other remaining four QTLs conferring agronomic traits were detected. Out of them, two (btnicl and btnic2) QTLs were detected for NIC, two (bttn1 and bttn2) for TN, three (btts1, btts2, and btts3) for TS, one (btph) for PH, one (btls) for LS, one (btpt) for PT and one ( $b t l)$ for L. However, no QTLs were detected for TK, LN, and W. The 11 main QTLs explained $12.3 \%$ to $26.4 \%$ of phenotypic variation of traits detected. Additionally, btnicl and bttnl respectively controlling NIC and TN showed a good co-segregation, indicating that there could be a certain unknown biological relationship between nicotine biosynthesis and nitrogen metabolism in tobacco leaf. The present study would provide a better understanding for the genetic control and further fine mapping of chemical components and agronomic traits in burley tobacco.
\end{abstract}

Keywords: Burley tobacco; Genetic linkage map; Agronomic traits; Genetic dissection

\footnotetext{
本研究由国家烟草专卖局项目(110200202004)资助。

“通讯作者(Corresponding author): 林国平, E-mail: lgpfy@126.com; Tel: 027-83641902

第一作者联系方式: E-mail: ccchun2001@yahoo.com.cn; Tel: 027-83606709

Received(收稿日期): 2009-02-22; Accepted(接受日期): 2009-04-29.
} 
烟草是经济作物的一种, 而且是最早应用于分 子生物学和基因工程的植物之一, 但目前烟草的基 础性研究十分薄弱, 因此, 加强烟草重要性状的遗 传控制机理研究, 以现代分子生物学育种手段培育 适应卷烟工业新形势发展所需的新品种, 降焦减害, 有力促进我国烟草育种科学的持续健康发展, 具有 十分重要的意义。

栽培种烟草(Nicotiana tabacum L.) 有 48 条染色 体 $(2 n=48)$, 基因组全长约 $4600 \mathrm{Mb}$, 约为人类基因 组全长的 1.5 倍 $^{[1]}$, 白胁烟属淡色晾烟, 是普通烟草 的缺绿变异型, 具有调和香气和吃味的作用, 是混 合型卷烟的重要原料。由于烟草的进化历史较短, 其遗传背景比较狭窄, 国外研究人员利用 RAPD 标 记 ${ }^{[2]}$ 和 AFLP 标记 ${ }^{[3-5]}$ 已经揭示了烟草较低的遗传多 样性, 因此, 目前被正式报道的烟草遗传连锁图谱 的数量较少, 标记密度有限, 影响了图谱质量和可 用性。Lin 等 ${ }^{[6]}$ 利用来自烟草野生种间杂交 $N$. plumbaginifolia $\times N$. Longiflora 所获得的 99 个 $\mathrm{F}_{2}$ 植株, 采用 69 个 RFLP 标记和 102 个 RAPD 标记构建了包 括 9 个主要连锁群、总长度为 $1062 \mathrm{cM}$ 的第一张烟 草分子标记遗传连锁图谱。Nishi 等 ${ }^{[7]}$ 利用 125 个白 胁烟 DH 系所组成的 DH 群体, 采用 117 个 AFLP 分 子标记构建了包含 10 个连锁群的第一张白胁烟遗 传图谱, 其总长为 $383 \mathrm{cM}$, 标记间平均遗传距离为 $3.3 \mathrm{cM}^{2} 2006$ 年肖炳光等 ${ }^{[8]}$ 利用由 137 个烤烟 $\mathrm{DH}$ 系组成的作图群体, 采用 10 个 ISSR 标记和 147 个 RAPD 标记构建了国内第一张烤烟分子遗传连锁图 谱, 包括 27 个连锁群, 总长度为 $1838.2 \mathrm{cM}$, 标记 间平均遗传距离为 $14.1 \mathrm{cM}^{\circ}$ 。肖炳光等 ${ }^{[9]}$ 在以前作图 数据的基础上新增 23 个标记构建了包括 11 个 ISSR 标记和 158 个 RAPD 标记、由 27 个连锁群组成的烤 烟分子标记遗传连锁图谱, 其覆盖长度达到了 $2094.6 \mathrm{cM}$, 是目前发表的最长的烟草遗传连锁图 谱。Julio 等 ${ }^{[10]}$ 利用 114 个烤烟重组自交系采用 ISSR、 AFLP 和 SSAP 等标记构建了一张烤烟分子遗传连锁 图谱, 该图谱共 18 个连锁群, 包括 138 个分子标记, 总长 $707.6 \mathrm{cM}$ 。Bindler 等 ${ }^{[11]}$ 利用烟草基因组计划 (tobacco genome initiative, TGI)中的基因组序列所开 发的 SSR 标记基于 186 个 $F_{2}$ 植株构建了一张烤烟的 分子遗传连锁图谱, 该图谱共 24 个连锁群, 293 个 $\mathrm{SSR}$ 标记, 总长为 $1920 \mathrm{cM}$ 。马红勃等 ${ }^{[12]}$ 利用由烤 烟和白胁烟杂交而来的 187 个 $\mathrm{F}_{2}$ 单株组成的作图群 体采用 SRAP 标记和 ISSR 标记构建了一张包含 26
个连锁群、 112 个标记、总长度为 $1560.2 \mathrm{cM}$ 的遗 传图谱。另一方面, 大多烟草研究是通过集团分离 分析法(bulked segregation analysis, BSA) 获得与抗 病基因, 如马铃薯 $\mathrm{Y}$ 病毒 $(\mathrm{PVY})^{[13]}$ 、黑根腐病 ${ }^{[14-15]}$ 、 黑胫病 ${ }^{[16]}$ 、根结线虫病 ${ }^{[17]}$ 、青枯病 ${ }^{[7]}$ 和野火病 ${ }^{[18]}$ 相 连锁的标记，而对于其他综合性状尤其是影响烟叶 质量的化学成分和农艺性状等研究比较少见。Julio 等 ${ }^{[10]}$ 利用其构建的烤烟遗传连锁图谱对包括农艺性 状、叶片质量、化学成分及卷烟特性等在内的 59 个 性状进行了 QTL 定位分析, 共检测到相关 QTL 75 个, 贡献率在 $8.0 \% \sim 41.5 \%$ 之间, 其中 19 个 QTL 表 现与双亲期望相反的效应。肖炳光等 ${ }^{[9]}$ 利用 DH 群体 对烤烟总糖、烟碱和氧化钾进行初步的 QTL 分析, 共 检测到 7 个相关 QTL，加性 QTL 的贡献率在 $1.27 \%$ $8.27 \%$ 之间。

国内对于白胁烟遗传连锁图谱的构建和重要性 状的 QTL 定位分析尚未见报道, 本研究旨在剖析白 胁烟烟叶主要化学成分和部分农艺性状的遗传控制 机理, 为进一步定位和克隆相关功能基因创造条件, 进而为白胁烟新品种选育提供理论依据。

\section{1 材料与方法}

\section{1 供试材料}

亲本材料 Burley $37\left(\mathrm{P}_{1}\right)$ 和 Burley $21\left(\mathrm{P}_{2}\right)$ 分别为 高、低烟碱含量的优质白胁烟品种, 目前普遍用于 白胁烟雄性不育系杂交育种。2004 年 7 月在湖北省 恩施烟草基地配制 $F_{1}\left(P_{1} \times P_{2}\right)$ 代, 2005 年 7 月中下旬 在中国烟草白胁烟试验站经组织培养及染色体加倍 技术获得 DH 群体 ${ }^{[19]}$, 共计 94 个 DH 系(包括两个亲 本 $P_{1}$ 和 $\left.P_{2}\right), 2006-2007$ 连续两年种植在湖北省建始 县三里坝乡。

\subsection{DNA 提取及标记分析}

采集亲本 Burley 37、Burley 21 和 DH 群体苗期 茁壮的叶片, 采用李佳等 ${ }^{[20]}$ 的方法提取总 DNA。

经初步篮选, 选取多态性较好的两组酶切组合, Sac I/Mse I 和 Pst I/Mse I, 分别篮选引物 825 对和 2100 对, 引物序列由上海生物工程技术服务有限公 司合成。总 DNA 的酶切、预扩、选扩反应体系及 PCR 扩增程序完全参照陆光远等 ${ }^{[21]}$ 的方法。

根据 $\mathrm{Li}$ 等 ${ }^{[22]}$ 提出的原则设计 SRAP 引物, 从中 选取 620 对, 由上海生物工程技术服务有限公司合 成。本研究所用 SRAP 的正、反向引物见表 1 。PCR 反应体系含带 $\left(\mathrm{NH}_{4}\right)_{2} \mathrm{SO}_{4}$ 的 $1 \times$ buffer、 $1.5 \mathrm{mmol} \mathrm{L}^{-1}$ 
表 1 本研究所使用的正、反向 SRAP 引物序列

Table 1 Sequences of SRAP forward and reverse primers used in the study

\begin{tabular}{cll}
\hline $\begin{array}{c}\text { 正向引物 } \\
\text { Forward primers (5'-3') }\end{array}$ & \multicolumn{1}{c}{$\begin{array}{c}\text { 正向引物 } \\
\text { Forward primers (5'-3') }\end{array}$} & $\begin{array}{c}\text { 反向引物 } \\
\text { Reverse primers (5'-3') }\end{array}$ \\
\hline ME1: TGAGTCCAAACCGGATA & ME17: TTCAGGGTGGCCGGATG & EM1: GACTGCGTACGAATTAAT \\
ME2: TGAGTCCAAACCGGAGC & ME18: TGGGGACAACCCGGCTT & EM2: GACTGCGTACGAATTTGC \\
ME3: TGAGTCCAAACCGGAAT & ME19: CTGGCGAACTCCGGATG & EM3: GACTGCGTACGAATTGAC \\
ME4: TGAGTCCAAACCGGACC & ME20: GGTGAACGCTCCGGAAG & EM4: GACTGCGTACGAATTTGA \\
ME5: TGAGTCCAAACCGGAAG & ME21: AGCGAGCAAGCCGGTGG & EM5: GACTGCGTACGAATTAAC \\
ME6: TGAGTCCAAACCGGTAG & ME22: GAGCGTCGAACCGGATG & EM6: GACTGCGTACGAATTGCA \\
ME7: TGAGTCCAAACCGGTTG & ME23: CAAATGTGAACCGGATA & EM7: GACTGCGTACGAATTATG \\
ME8: TGAGTCCAAACCGGTGT & ME24: GAGTATCAACCCGGATT & EM8: GACTGCGTACGAATTAGC \\
ME9: TGAGTCCAAACCGGTCA & ME25: GTACATAGAACCGGAGT & EM9: GACTGCGTACGAATTACG \\
ME10: TGAGTCCAAACCGGTAC & ME26: TACGACGAATCCGGACT & EM10: GACTGCGTACGAATTTAG \\
ME11: TGAGTCCAAACCGGATG & ME27: CACAGTCATGCCGGAAT & EM11: GACTGCGTACGAATTTCG \\
ME12: TGAGTCCAAACCGGACA & ME28: GACCAGTAAACCGGATG & EM12: GACTGCGTACGAATTGCT \\
ME13: TGAGTCCAAACCGGGAT & ME29: CAGGACTAAACCGGATA & EM13: GACTGCGTACGAATTGGT \\
ME14: TGAGTCCAAACCGGGCT & ME30: ATCAGTCGGACCGGATT & EM14: GACTGCGTACGAATTCAG \\
ME15: TGAGTCCAAACCGGTAA & ME31: GATTGCATCACCGGATG & EM15: GACTGCGTACGAATTCTG \\
ME16: TGAGTCCAAACCGGTGC & & EM16: GACTGCGTACGAATTCGG \\
& & EM17: GACTGCGTACGAATTCCA \\
& & EM18: GACTGCGTACGAATTCAA \\
\end{tabular}

$\mathrm{MgCl}_{2} 、 200 \mu \mathrm{mol} \mathrm{L}{ }^{-1} \mathrm{dNTPs} 、 1 \mathrm{U} \mathrm{Taq}$ 酶(购自 MBI 公司)、100 ng 模板 DNA、正向和反向引物各 $50 \mathrm{ng}$, 用 $\mathrm{ddH}_{2} \mathrm{O}$ 补足总体积 $20 \mu \mathrm{L}$ 。 $\mathrm{PCR}$ 反应程序为 $94^{\circ} \mathrm{C}$ $1 \mathrm{~min} ; 35^{\circ} \mathrm{C} 1 \mathrm{~min}, 72^{\circ} \mathrm{C} 1 \mathrm{~min}$, 共 5 个循环; $94^{\circ} \mathrm{C} 1$ min, $50^{\circ} \mathrm{C} 1 \mathrm{~min}, 72^{\circ} \mathrm{C} 1 \mathrm{~min}$, 共 35 个循环; $4^{\circ} \mathrm{C}$ 保 存。在 PTC-225 型热循环仪(MJR, 美国)上进行 PCR 反应。

以上两种引物的 PCR 扩增产物(除 AFLP预扩产 物外)均用聚丙烯酰胺凝胶 $\left(6 \%, 7 \mathrm{~mol} \mathrm{~L}^{-1}\right)$ 电泳分离, 电泳所用仪器为 Sequi-Gen sequencing cell (Bio-Rad,

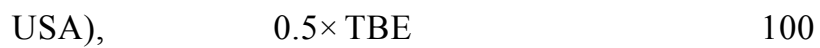
$\mathrm{W}$ 预电泳 $30 \mathrm{~min}$ 左右(玻璃板面温度约为 $50^{\circ} \mathrm{C}$ ), 上 样后, 用恒定功率 $85 \mathrm{~W}$ 电泳 $1.0 \sim 1.5 \mathrm{~h}$, 参照陆光远 等 ${ }^{[21]}$ 的方法进行电泳后的染色与显影。

\section{3 遗传连锁图谱构建}

采用 Mapmaker/Exp, Ver. 3.0 $0^{[23]}$, 选用 Kosambi 函数将重组值转换为图距单位 (cM)。先用“ group” 命令对所有标记进行分组 $(L O D \geq 4.0)$, 将标记分配 到不同连锁群, 对少于 5 个标记的连锁群直接运用

“compare” 命令排序, 多于 5 个标记的连锁群, 运
用“ three point” 命令进行多点测验, 运用“ order” 命令进行标记之间排序，对于多个标记同时出现在 一个可能位置, 运用“ try” 命令, 将标记逐个插入到 适合的位置, 然后运用“ ripple” 命令检验连锁群中 标记的最佳顺序。用“ map” 命令构建连锁图。采用

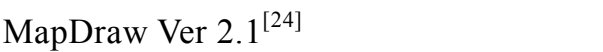

\section{4 分子标记数据统计、命名}

对于显性标记, 亲本及群体单株根据带型有无 记为 1 或 0 ; 对于共显性标记, 亲本 Burley 37 的带 型记为 $\mathrm{A}$, 亲本 Burley 21 的带型记为 $\mathrm{B}$ ，凡与亲本 Burley 37 带型一致的单株带型记为 $\mathrm{A}$, 凡与亲本 Burley 21 带型一致的单株带型记为 B, 缺失数据记 为 -”。采用引物组合的方法对标记进行命名。SRAP 标记与 AFLP 标记用原始的标记名称, 如 EM10ME03 表示 SRAP 正向引物 EM10 与反向引物 ME03 的组合, P10-M03 表示 AFLP 引物 P10 与 M03 的 组合。

\section{5 田间试验设计}

单行区种植, 每小区种植 10 株, 行株距 $120 \mathrm{~cm} \times$ $45 \mathrm{~cm}$, 四周设保护行。完全随机区组设计, 3 次重复, 
以亲本 Burley 37 和 Burley 21 作对照, 相邻种植, 每 隔 20 小区种一对照。按当地优质烟叶生产技术规范 进行田间管理。

\section{6 农艺性状调查及样品分析}

烟株打顶后, 每小区随机选取 5 株, 观察记载 被选 DH 系的株高、茎围、节距、总叶数、中部叶 的长和宽等性状。在晾制结束后, 每小区随机选取 2 株, 取 4 片中部叶, 叶位为 $12 、 13$ 叶位(自下而上), 在鼓风干燥箱内烘干并磨碎制成分析样本 ${ }^{[25]}$ 。参照 中华人民共和国烟草行业标准 YC/T160-2002 [26]采 用连续流动分析仪(API，美国)分析烟碱、总氮、总 钾、总糖等化学成分。

\subsection{QTL 定位分析与命名}

利用 Windows QTL Cartographer Ver. 2.5 软件 ${ }^{[27]}$, 结合性状表型数据和遗传连锁图谱结果进行 QTL 定 位分析。采用 Modle6(考虑区间 $10 \mathrm{cM}$ 内的 5 个最 大 $F$ 值的辅助因子), 在 $P=0.05$ 的显著水平下, 排步 测验 1000 次后, 确定 $\mathrm{LOD} \geq 3$ 作为检测性状 QTL 的阈值。各 DH 系性状表型数据取两年均值, 其中烟 碱、总氮、总糖、总钾等化学成分取晾制后中部烟 叶两年的均值。运行软件的结果可同时给出 QTL 的 加性效应和贡献率等。

QTL 的命名按烟草类型 (本研究白胁烟为 $b t)+$ 性 状名称(如烟碱为 $n i c$, 总氮为 $t n$, 总糖为 $t s$, 总钾为 $t k$, 株高为 $p h$, 茎围为 $l s$, 节距 $p t$, 总叶数 $l n$, 中部 叶长为 $l$, 中部叶宽为 $w$ 等)+序号(相同性状的两个 以上 QTL 位点按 $1,2,3 \cdots$ 依次编写)。

\section{2 结果与分析}

\section{1 分子标记分析}

以亲本 Burley 37 和 Burley 21 为材料篮选引物, 将获得的稳定、清晰可靠的多态性引物在 DH 群体 中进行检测。共篮选 AFLP 引物 2925 对, 获得在亲 本间多态性明显且条带清晰易读的引物 72 对, 在 DH 群体中检测到标记 139 个, 多态性比例为 1.9 ; 共篮 选 SRAP 引物 620 对, 其中 5 对引物表现较好, 扩展
出标记 8 个, 多态性比例为 1.6 。对所有标记的分离 比进行 $\chi^{2}$ 检验, 在 $P=0.01$ 水平上有 25 个 AFLP 标 记表现偏分离, 占标记总数的 $17.0 \%$, 其中 18 个标 记偏向亲本 Burley 37(72.0\%), 另外 7 个标记偏向亲 本 Burley 21 (28.0\%)(表 2)。

\section{2 遗传连锁图谱的构建}

利用篮选获得的 147 个分子标记构建白胁烟遗 传连锁图谱, 其中 118 个分子标记包括 112 个 AFLP 标记和 6 个 SRAP 标记构成了 22 个连锁群(A1 A22), 另外 29 个分子标记包括 27 个 AFLP 和 2 个 SRAP 标记未能连锁到图谱上(表 2)。该图谱总遗传距离为 $1953.6 \mathrm{cM}$, 连锁群的遗传距离从最短 $0.5 \mathrm{cM}$ 到最 长的 $191.9 \mathrm{cM}$, 标记间的平均距离为 $20.5 \mathrm{cM}$, 最短 为 $0.5 \mathrm{cM}$, 最长为 $44.9 \mathrm{cM}$ 。连锁群上的标记数目从 最少 2 个到最多 12 个, 偏分离标记主要集中在第 1 、 11 和 14 连锁群上, 而有 10 个连锁群没有偏分离标 记(表 3)。

\section{3 部分性状的 QTL 分析}

2.3.1 亲本和 $\mathrm{DH}$ 群体的性状表现利用最小显 著差数法(least significance difference, $L S D$ ) 检测两 亲本性状表现的显著性, 结果表明, 除烟碱双亲间 存在显著差异外 $(P<0.05)$, 其他性状双亲间均不存 在显著差异 $(P>0.05)$ (表 4$)$ 。从极值范围来看, 各性 状均存在不同程度的双向超亲现象。从 DH 群体的 斜度和峰度指标来看, 除了节距轻度偏离正态分布 之外, 其他性状均比较符合正态分布, 说明这些性 状具备数量性状特征。对各性状进行二因素(年份与 基因型)方差分析表明(表略), 4 种化学成分中除总钾 在年份和基因型间均不存在显著差异外, 其他 3 种 化学成分(烟碱、总氮、总糖)年份和基因型间均存在 显著差异, 其中烟碱在年份和基因型间达到极显著 差异, 农艺性状除株高和总叶数在基因型间存在显 著差异外，在年份和基因型间均不存在显著差异。

2.3.2 部分性状的 QTL 定位分析 共检测到 11 个主效 QTL 与各主要性状显著相关, 涉及到 7 个连 锁群(A1、A4、A5、A6、A8、A10 和 A17), 其中第

表 2 构建遗传连锁图谱的分子标记分析

Table 2 Analysis of molecular markers constructing genetic linkage map

\begin{tabular}{ccccccc}
\hline $\begin{array}{c}\text { 标记类型 } \\
\text { Type }\end{array}$ & $\begin{array}{c}\text { 引物数 } \\
\text { No. of primers }\end{array}$ & $\begin{array}{c}\text { 多态性标记数 } \\
\text { No. of polymorphic loci }\end{array}$ & $\begin{array}{c}\text { 多态性比例 } \\
\text { Polymorphism } \\
\text { ratio }\end{array}$ & $\begin{array}{c}\text { 偏分离标记数 } \\
\text { No. of distortion- } \\
\text { segregation loci }\end{array}$ & $\begin{array}{c}\text { 连锁标记数 } \\
\text { No. of linked } \\
\text { loci }\end{array}$ & $\begin{array}{c}\text { 末连锁标记数 } \\
\text { No. of unlinked } \\
\text { loci }\end{array}$ \\
\hline AFLP & 72 & 139 & 1.9 & 25 & 27 \\
SRAP & 5 & 8 & 1.6 & 0 & 2 \\
合计 Total & 77 & 147 & & 25 & 118 \\
\hline
\end{tabular}


表 3 连锁群上遗传距离和标记分布

Table 3 Distribution and genetic distance of markers in the linkage groups

\begin{tabular}{|c|c|c|c|c|c|c|}
\hline $\begin{array}{c}\text { 连锁群 } \\
\text { Linkage group }\end{array}$ & $\begin{array}{l}\text { 长度 } \\
\text { Length } \\
(\mathrm{cM})\end{array}$ & $\begin{array}{c}\text { 标记数 } \\
\text { No. of markers }\end{array}$ & $\begin{array}{c}\text { 偏分离标记数 } \\
\text { No. of distortion- } \\
\text { segregation markers }\end{array}$ & $\begin{array}{c}\text { 最大标记间距 } \\
\text { Max interval } \\
\text { (cM) }\end{array}$ & $\begin{array}{c}\text { 最小标记间距 } \\
\text { Min interval } \\
\text { (cM) }\end{array}$ & $\begin{array}{c}\text { 平均图距 } \\
\text { Average interval } \\
\text { (cM) }\end{array}$ \\
\hline A1 & 99.1 & 12 & 4 & 13.5 & 5.1 & 9.0 \\
\hline A2 & 0.5 & 2 & 0 & 0.5 & 0.5 & 0.5 \\
\hline $\mathrm{A} 3$ & 73.0 & 5 & 1 & 22.9 & 9.9 & 18.3 \\
\hline A4 & 99.0 & 8 & 2 & 17.3 & 12.1 & 14.1 \\
\hline A5 & 15.1 & 2 & 0 & 15.1 & 15.1 & 15.1 \\
\hline A6 & 68.0 & 5 & 0 & 20.8 & 13.1 & 17.0 \\
\hline A7 & 120.6 & 7 & 2 & 21.4 & 18.5 & 20.1 \\
\hline A 8 & 99.9 & 5 & 1 & 33.3 & 21.7 & 25.0 \\
\hline A9 & 43.3 & 4 & 0 & 20.5 & 5.3 & 14.4 \\
\hline A 10 & 177.7 & 8 & 2 & 38.3 & 20.2 & 25.4 \\
\hline A11 & 191.9 & 8 & 4 & 44.9 & 19.3 & 27.4 \\
\hline A12 & 137.1 & 6 & 1 & 30.5 & 24.1 & 27.4 \\
\hline A13 & 187.4 & 7 & 2 & 43.0 & 27.2 & 31.2 \\
\hline A14 & 131.8 & 8 & 3 & 23.6 & 12.1 & 18.8 \\
\hline A15 & 91.2 & 6 & 2 & 23.2 & 7.9 & 18.2 \\
\hline A16 & 101.7 & 6 & 0 & 33.5 & 11.2 & 20.3 \\
\hline A 17 & 110.4 & 6 & 1 & 25.8 & 16.9 & 22.1 \\
\hline A 18 & 19.2 & 2 & 0 & 19.2 & 19.2 & 19.2 \\
\hline A19 & 57.6 & 3 & 0 & 29.0 & 28.6 & 28.8 \\
\hline $\mathrm{A} 20$ & 48.4 & 3 & 0 & 27.4 & 21.0 & 24.2 \\
\hline A 21 & 53.1 & 3 & 0 & 26.8 & 26.3 & 26.6 \\
\hline A 22 & 27.6 & 2 & 0 & 27.6 & 27.6 & 27.6 \\
\hline 合计 Total & 1953.6 & 118 & 25 & 44.9 & 0.5 & 20.5 \\
\hline
\end{tabular}

表 4 亲本和 DH 群体性状描述性统计

Table 4 Descriptive statistics of traits for the parents and the DH population

\begin{tabular}{|c|c|c|c|c|c|c|}
\hline \multirow{2}{*}{$\begin{array}{l}\text { 性状 } \\
\text { Trait }\end{array}$} & \multicolumn{2}{|c|}{ 亲本 Parent $($ mean $\pm S D)$} & \multicolumn{4}{|c|}{ DH 群体 DH population } \\
\hline & Burley 37 & Burley 21 & $\begin{array}{c}\text { 平均数土标准差 } \\
\text { Mean } \pm S D\end{array}$ & $\begin{array}{l}\text { 范围 } \\
\text { Range }\end{array}$ & $\begin{array}{c}\text { 斜度 } \\
\text { Skewness }\end{array}$ & $\begin{array}{c}\text { 峰度 } \\
\text { Kurtosis }\end{array}$ \\
\hline 烟碱 Nicotine (\%) & $4.66 \pm 0.78 \mathrm{a}$ & $3.23 \pm 0.45 \mathrm{~b}$ & $3.41 \pm 0.67$ & $1.38-5.59$ & 0.24 & -0.29 \\
\hline 总氮 Total nitrogen (\%) & $5.23 \pm 0.70$ & $4.96 \pm 0.57$ & $5.37 \pm 0.84$ & $3.99-7.10$ & -0.46 & 0.78 \\
\hline 总糖 Total sugar (\%) & $0.44 \pm 0.05$ & $0.42 \pm 0.06$ & $0.52 \pm 0.01$ & $0.28-0.72$ & 0.12 & 0.07 \\
\hline 总钾 Total potassium (\%) & $4.39 \pm 0.34$ & $3.69 \pm 0.31$ & $3.90 \pm 0.05$ & $2.82-5.12$ & 0.62 & 0.32 \\
\hline 株高 Plant height (cm) & $155.00 \pm 3.12$ & $156.70 \pm 2.11$ & $145.60 \pm 9.10$ & $121.50-166.80$ & -0.28 & 0.21 \\
\hline 茎围 Stalk circumference (cm) & $9.80 \pm 0.10$ & $10.90 \pm 0.21$ & $10.40 \pm 0.79$ & $8.80-12.80$ & 0.58 & 0.88 \\
\hline 节距 Distance between nodes $(\mathrm{cm})$ & $4.30 \pm 0.09$ & $3.80 \pm 0.11$ & $4.20 \pm 0.43$ & $3.50-5.80$ & 1.05 & 1.72 \\
\hline 总叶数 Total No. of leafs (cm) & $30.00 \pm 0.23$ & $31.20 \pm 0.18$ & $30.00 \pm 1.17$ & $27.00-32.70$ & -0.11 & -0.07 \\
\hline 中部叶长 Length of central leaf $(\mathrm{cm})$ & $72.00 \pm 0.41$ & $72.80 \pm 0.34$ & $69.10 \pm 4.33$ & $56.20-77.80$ & -0.44 & 0.15 \\
\hline 中部叶宽 Width of central leaf (cm) & $30.30 \pm 0.17$ & $31.70 \pm 0.13$ & $31.80 \pm 2.40$ & $26.50-37.80$ & 0.40 & 0.11 \\
\hline
\end{tabular}

$\mathrm{a}$ 和 $\mathrm{b}$ 表示亲本间在 0.05 水平上差异显著。

a, b: significantly different between parents at $P<0.05$.

10 连锁群聚集了 5 个 QTL, 第 1 连锁群聚集了 4 个 QTL(图 1)。其中与烟碱和总氮相关的 QTL 各 2 个, 总糖相关的 QTL 有 3 个, 与株高、茎围、节距和中 部叶长相关的 QTL 各有 1 个, 没有检测到与中部叶
宽、总叶数、总钾相关的 QTL(表 5)。

控制烟碱的 2 个主效 QTL (btnicl 和 btnic2) 分别 位于第 1 和第 10 连锁群, 二者贡献率相近, 分别为 $16.7 \%$ 和 $17.0 \%$ ，二者的加性效应分别来自 Burley 21 

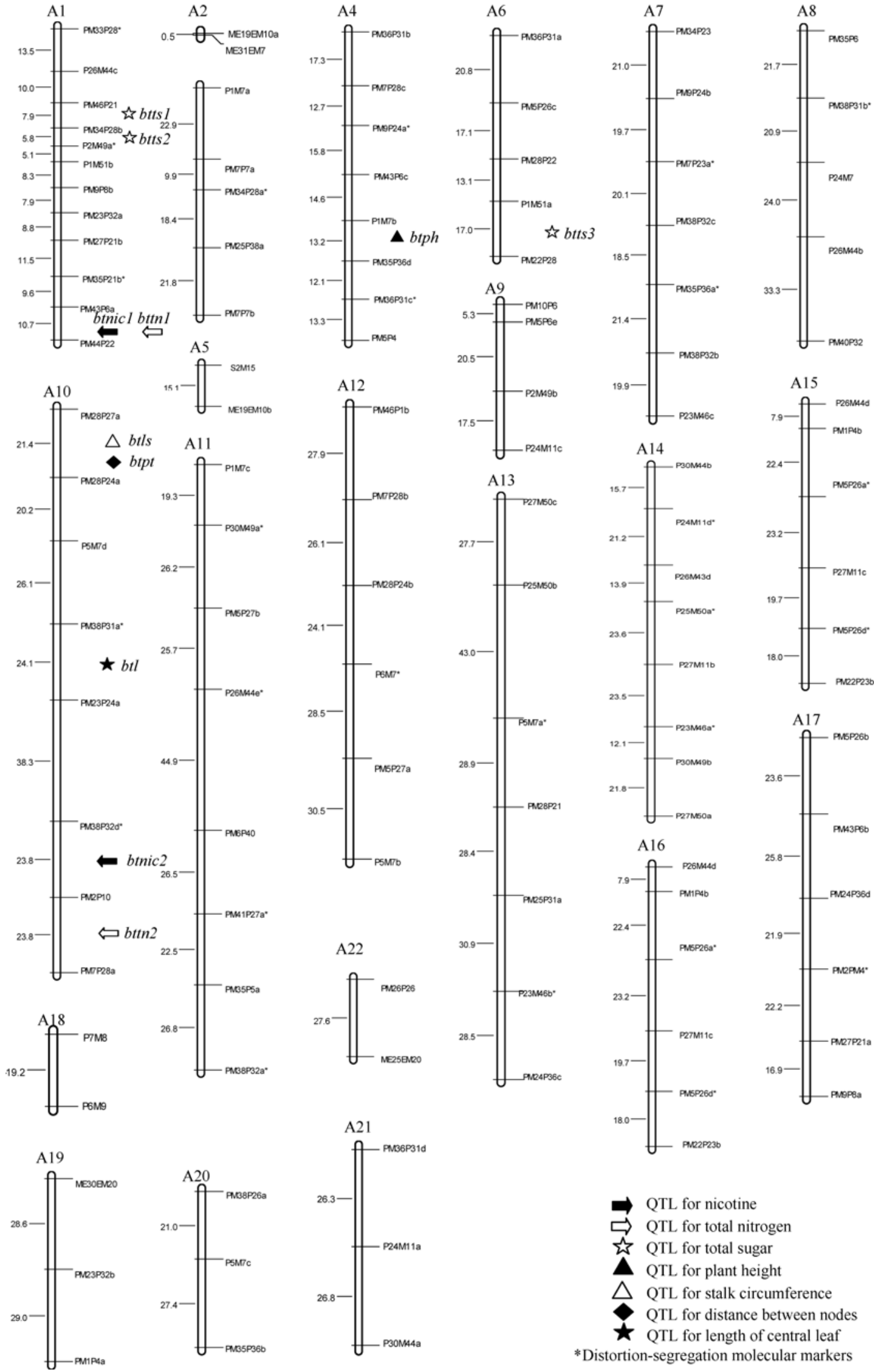

QTL for nicotine

$\Rightarrow$ QTL for total nitrogen

QTL for total sugar

ATL for plant height

$\triangle$ QTL for stalk circumference

QTL for distance between nodes QTL for length of central leaf * Distortion-segregation molecular markers

图 1 白肋烟遗传连锁图谱及部分性状 QTL

Fig. 1 Genetic linkage map and QTLs controlling partial traits of burley tobacco 
表 5 白肋烟 DH 群体部分性状的 QTL

Table 5 QTLs of partial traits in the DH population of burley tobacco

\begin{tabular}{|c|c|c|c|c|c|c|c|c|c|}
\hline $\begin{array}{l}\text { 性状 } \\
\text { Trait }\end{array}$ & $\begin{array}{l}\text { 连锁群 } \\
\text { Group }\end{array}$ & QTL & $\begin{array}{c}\text { 标记区间 } \\
\text { Flanking markers }\end{array}$ & $\begin{array}{c}\text { 置信区间 } \\
\text { Trust interval } \\
(\mathrm{cM}) \\
\end{array}$ & $\begin{array}{c}\text { 峰值 } \\
\text { Peak posi- } \\
\text { tion (cM) }\end{array}$ & $L O D$ & $\begin{array}{c}\text { 加性效应 } \\
\text { Additive } \\
(\%)\end{array}$ & $\begin{array}{c}\text { 贡献亲本 } \\
\text { Contribution } \\
\text { parent } \\
\end{array}$ & $\begin{array}{c}\text { 贡献率 } \\
R^{2}(\%)\end{array}$ \\
\hline \multirow{2}{*}{ 烟碱 NIC } & A1 & btnicl & PM43P06a-PM44P22 & $93.8-98.4$ & 98.40 & 4.70 & -0.56 & Burley 21 & 16.7 \\
\hline & $\mathrm{A} 10$ & btnic2 & РM38P32-PM02P10 & $143.9-168.1$ & 153.90 & 4.90 & 0.47 & Burley 37 & 17.0 \\
\hline \multirow{2}{*}{ 总氮 TN } & $\mathrm{A} 1$ & $b t t n 1$ & PM43P06a-PM44P22 & $94.8-98.4$ & 98.40 & 3.92 & -0.39 & Burley 21 & 14.6 \\
\hline & $\mathrm{A} 10$ & $b t t n 2$ & PM02P10-PM07P28a & $143.8-168.1$ & 157.90 & 3.48 & 0.33 & Burley 37 & 16.1 \\
\hline \multirow{3}{*}{ 总糖 TS } & A1 & $b t t s 1$ & PM46P21-PM34P28b & $94.8-98.4$ & 27.50 & 4.10 & -0.85 & Burley 21 & 22.1 \\
\hline & $\mathrm{A} 1$ & $b t t s 2$ & PM34P28b-P2M49a & $143.8-168.1$ & 33.40 & 3.16 & -0.73 & Burley 21 & 17.3 \\
\hline & A6 & $b t t s 3$ & P1M51a-PM22P28 & $23.8-31.4$ & 59.00 & 3.11 & -0.54 & Burley 21 & 18.3 \\
\hline 株高 PH & A4 & btph & P1M7b-PM35P36d & $62.6-78.4$ & 70.40 & 4.60 & 32.38 & Burley 37 & 26.4 \\
\hline 茎围 LS & $\mathrm{A} 10$ & $b t l s$ & PM28P27a-PM28P24a & $0-14.0$ & 0.01 & 3.04 & 1.68 & Burley 37 & 12.3 \\
\hline 节距 PT & A10 & $b t p t$ & PM28P27a-PM28P24a & $0-9.9$ & 0.01 & 3.92 & 0.91 & Burley 37 & 17.0 \\
\hline 中部叶长 L & $\mathrm{A} 10$ & $b t l$ & PM38P31a-PM23P24a & $57.6-91.7$ & 69.70 & 3.26 & 8.03 & Burley 37 & 16.2 \\
\hline
\end{tabular}

表中负的加性效应代表该效应是由亲本 Burley 21 贡献的，正的加性效应代表该效应是由亲本 Burley 37 贡献的。

A positive additive effect is from high nicotine content parent Burley 37, a negative additive effect is from low nicotine content parent Burley 21. NIC: nicotine, TN: total nitrogen, TS: total sugar, PH: plant height, LS: stalk circumference, PT: distance between nodes, L: length of central leaf.

和 Burley 37, Burley 21 在 btnicl 位点的等位基因使 烟碱降低了 $0.56 \%$ 。Burley 37 在 btnic2 位点的等位 基因提高了 $0.47 \%$ 的烟碱; 与晾制后中部叶总氮相 关的主效 QTL 有 2 个, $b t t n 1$ 和 $b t t n 2$, 分别位于第 1 和第 10 连锁群上, 贡献率分别为 $14.6 \%$ 和 $16.1 \%$, 加 性效应分别使总氮降低和提高了 $0.39 \%$ 和 $0.33 \%$, btnic1 与 bttn1 处于相同的标记区间, 且峰值相同, 处于共分离状态; 与总糖相关的 QTL 有 3 个, btts 1 、 $b t t s 2$ 和 $b t t s 3$, 前两者均分布于第 1 连锁群, 第 3 个 分布于第 6 连锁群, 贡献亲本均为 Burley 21 , 分别 降低了 $0.85 \% 、 0.73 \%$ 和 $0.54 \%$ 的总糖, 贡献率分别 为 $22.1 \% 、 17.3 \%$ 和 $18.3 \%$; 与株高相关的 QTL btph 被定位在第 4 连锁群, 处于 AFLP 标记 P1M7b 与 PM35P36d 之间, 其贡献率为 $26.4 \%$, 为所有 QTL 中 最大贡献率者, 亲本 Burley 37 使株高增加了 32.38 $\mathrm{cm}$; 在第 10 连锁群上检测到 $b t l s, b t p t$ 和 $b t l 3$ 个 QTL, 分别影响茎围、节距和中部叶长的表现, 其中 $b t l s$ 和 btpt 均处于 AFLP 标记 PM28P27a PM28P24a 之 间, 峰值相同, 贡献率分别为 $12.3 \%$ 和 $17.0 \%, b t l$ 对 中部叶长的贡献率为 $16.2 \%$, 亲本 Burley 37 在这 3 个 QTL 位点处的等位基因分别使茎围、节距和中部 叶长增加了 $1.68 、 0.91$ 和 $8.03 \mathrm{~cm}$ (表 5)。

\section{3 讨论}

\section{1 白肋烟分子标记遗传连锁图谱}

烟草狭窄的遗传背景导致其分子标记的多态性 不丰富, 因此, 目前已构建的分子遗传图谱数量较
少 ${ }^{[6-12]}$, 且因使用的标记不同而可比性不强, 利用的 作图群体类型有 $\mathrm{F}_{2}$ 群体、DH 群体和重组自交系群 体, 分子标记类型主要包括 RFLP、SSR、AFLP、 RAPD、SRAP 及 ISSR 等, 图谱中标记总数从 117 至 293 个不等, 连锁群数目在 10 27 个之间, 图谱总 遗传距离最长为 $2094.6 \mathrm{cM}^{[9]}$, 最短仅 $383 \mathrm{cM}^{[7]}$ 。本 研究所构建的白胁烟遗传连锁图谱总遗传长度为 $1953.6 \mathrm{cM}$, 仅次于肖炳光等 ${ }^{[9]}$ 构建的烤烟连锁图谱 长度, 并采用了适合用来构建遗传连锁图谱的 SRAP 标记 ${ }^{[28]}$ 。目前烟草遗传连锁图谱的质量(标记数量和 密度)相对于其巨大的基因组而言仍然远远不够，仍 需要通过烟草基因组计划(TGI)发表的大量 EST 序 列、烟草 cDNA 文库、BAC 文库和其他茄科植物的 标记来补充大量的分子标记以不断增加图谱的饱和 度, 同时需适当增加作图群体大小。

\section{2 白肋烟性状的超亲现象}

超亲现象的产生一方面可能是双亲中控制性状 两个表型方向极值的基因分离表达的结果, 另一方 面也可能是隐性基因的纯合效应所致。关于第二点, Edwards 等 ${ }^{[29]}$ 在分析引起玉米的超亲现象的原因时 曾有过讨论。因此，我们可以充分利用超亲现象并 借助分子标记辅助选择手段获得性状表现极高或极 低的单株, 进而在遗传和育种中加以利用, 如选择 极低烟碱含量的 DH 系培育低烟碱品种等。

\section{3 白胁烟性状的 QTL 分析}

有关烟草重要性状的 QTL 定位分析研究报道较 少。Julio 等 ${ }^{[10]}$ 利用烤烟重组自交系群体扫描到与中 
部叶长相关的 QTL Dev3，贡献率为 9.2\%，控制烟碱 的 QTL NIRSTAR, 贡献率为 $13.3 \%$ 。肖炳光等 ${ }^{[9]}$ 利用 烤烟 DH 群体扫描到控制总糖、烟碱和氧化钾的加 性 QTL 分别为 2 个、 2 个和 3 个, 总贡献率分别为 $5.83 \% 、 9.54 \% 、 14.64 \%$ 。本研究扫描到与中部叶长 相关的主效 QTL 1 个, 贡献率 $16.2 \%$, 与烟碱和总糖 相关的主效 QTL 分别有 2 个和 3 个，总贡献率分别 为 $33.7 \%$ 和 $57.7 \%$, 没有扫描到与总钾、总叶数和中 部叶宽相关的 QTL。另外, 本文作者利用该 DH 群 体对白胁烟烟碱含量所作的遗传分析表明, 烟碱含 量主要受 2 对主基因控制, 与本研究结果较为一致 (尚未公开发表的数据)。

本研究检测到控制烟碱和总氮的 QTL(分别为 btnicl 和 bttn1)与一侧的 AFLP 标记 PM44P22 的遗 传距离仅为 $0.69 \mathrm{cM}$, 此标记可经进一步确认之后转 化为 SCAR 标记, 为用于分子标记辅助选择适宜烟 碱含量的白胁烟新品种提供了一种可能, 从而大大 提高育种的精确性，节省时间。另外, btnicl 和 bttn1 的共定位表明烟碱的合成和氮素吸收之间可能存在 某种目前尚不清楚的生物学相关性, 而且, 烟碱含 量与总氮含量也存在极显著的正相关 $(r=0.39, P<$ 0.001)。

本研究所构建的遗传连锁图谱为不同烟草类型 遗传图谱的相互比较、整合及高密度遗传连锁图谱 的构建和比较基因组学研究的开展提供了良好的参 考材料, 也为进一步精细定位和克隆控制白胁烟重 要性状的功能基因奠定了良好基础, 目前后续研究 工作正在进行中。

\section{4 结论}

构建了包括 112 个 AFLP 标记和 6 个 SRAP 标 记、由 22 个连锁群组成的白肋烟分子标记遗传连锁 图, 总遗传长度为 $1953.6 \mathrm{cM}$, 平均标记间距为 20.5 $\mathrm{cM}$ 。共检测到 11 个主效 QTL 可解释各性状表型变 异的 $12.3 \%$ 26.4\%, 其中 7 个与白胁烟晾制后中部 烟叶的烟碱、总氮、总糖等化学成分相关, 另外 4 个与株高、茎围、节距和中部叶宽等农艺性状相关。 控制烟碱和总氮的 QTL(分别为 btnicl 和 bttn 1) 处于 共分离状态，与一侧的 AFLP 标记 PM44P22 的遗传 距离仅为 $0.69 \mathrm{cM}$, 经转化为 $\mathrm{SCAR}$ 标记后对于分子 标记辅助育种有一定的利用价值。

\section{References}

[1] Galbraith D W, Harkins K R, Maddox J M, Ayres N M, Sharma D
P, Firoozabady E. Rapid flow cytometric analysis of the cell cycle in intact plant tissues. Science, 20: 1049-1051

[2] Del Piano L, Abet M, Sorrentino C, Acanfora F, Di Muro A. Genetic variability in Nicotiana tabacum and Nicotiana species as revealed by RAPD markers: 1. Development of the RAPD procedure. Beiträge zur Tabakforschung International (Contributions to Tobacco Research), 2000, 19: 1-15

[3] Julio E, Verrier J L, Dorlhac de Borne F. Development of SCAR markers linked to three disease resistances based on AFLP within Nicotiana tabacum L. Theor Appl Genet, 2006, 112: 335-346

[4] Ren N, Timko M P. AFLP analysis of genetic polymorphism and evolutionary relationships among cultivated and wild Nicotiana species. Genome, 2001, 44: 559-571

[5] Rossi L, Bindler G, Pijnenburg H, Isaac P G, Giraud-Henry I, Mahe M, Orvain C, Gadani F. Potential of molecular marker analysis for variety identification in processed tobacco. Plant Var Seeds, 2001, 14: 89-101

[6] Lin T Y, Kao Y Y, Lin S, Lin R F, Chen C M, Huang C H, Wang C K, Lin Y Z, Chen C C. A genetic linkage map of Nicotiana plumbaginifolia/Nicotiana longiflora based on RFLP and RAPD markers. Theor Appl Genet, 2001, 103: 905-911

[7] Nishi T, Tajima T, Noguchi S, Ajisaka H, Negishi H. Identification of DNA markers of tobacco linked to bacterial wilt resistance. Theor Appl Genet, 2003, 106: 765-770

[8] Xiao B-G(肖炳光), Xu Z-L(徐照丽), Chen X-J(陈学军), Shen A-R(申爱荣), Li Y-P(李永平), Zhu J(朱军). Genetic linkage map constructed by using a DH population for the flue-cured tobacco. Acta Tabacaria Sin (中国烟草学报)，2006，12(4)：35-40 (in Chinese with English abstract)

[9] Xiao B-G(肖炳光)， $\mathrm{Lu}$ X-P(卢秀萍)，Jiao F-C(焦芳蝉)， Li Y-P(李永平), Sun Y-H(孙玉合), Guo Z-K(郭兆奎). Preliminary QTL analysis of several chemical components in flue-cured tobacco (Nicotiana tabacum L.). Acta Agron Sin (作物学报), 2008, 34(10): 1762-1769 (in Chinese with English abstract)

[10] Julio E, Denoyes-Rothan B, Verrier J L, Dorlhac de Borne F. Detection of QTLs linked to leaf and smoke properties in Nicotiana tabacum based on a study of 114 recombinant inbred lines. Mol Breed, 2006, 18: 69-91

[11] Bindler G, Rutger van der Hoeven, Gunduz I, Plieske J, Ganal M, Rossi L, Gandani F, Donini P. A microsatellite marker based linkage map of tobacco. Theor Appl Genet, 2007, 114: 341-349

[12] Ma H-B(马红勃), Qi J-M(祁建民), Li Y-K(李延坤), Liang J-X(梁景霞), Wang $\mathrm{T}$ (王涛), Lan $\mathrm{T}$ (兰涛), Chen S-H(陈顺辉), Tao A-F(陶爱芬), Lin L-H(林荔辉), Wu J-M(吴建梅). Construction of a molecular genetic map of tobacco based on SRAP and ISSR markers. Acta Agron Sin (作物学报), 2008, 34(11): 19581963 (in Chinese with English abstract)

[13] Noguchi S, Tajima T, Yamamoto Y, Ohno T, Kubo T. Deletion of a large genomic segment in tobacco varieties that are resistant to potato virus Y (PVY). Mol Gen Genet, 1999, 262: 822-829

[14] Bai D, Brandle J E. Identification of two RAPD markers tightly linked with the Nicotiana debneyi for resistance to black root rot 
of tobacco. Theor Appl Genet, 1995, 91: 1184-1189

[15] Julio E, Verrier J L, Dorlhac de Borne F. Development of SCAR markers linked to three disease resistances based on AFLP within Nicotiana tabocum L. Theor Appl Genet, 2006, 112: 335-346

[16] Jonhson E S, Wernsman E A. Origin of the black shank resistance gene, $P h$, in tobacco cultivar Coker 371-Gold. Plant Dis, 2002, 86: 1080-1084

[17] Yi H Y, Rufty R C, Wernsman E A. Mapping the root-knot nematode resistance gene $(R k)$ in tobacco with RAPD markers. Plant Dis, 1998, 82: 1319-1322

[18] Yi Y H. RAPD markers elucidate the origin of the root-knot nematode resistance gene $(R k)$ in tobacco. Tob Sci, 1998, 42: 58-63

[19] Chai L-G(柴利广), Zhang J-J(张俊杰), Lin G-P(林国平), Wang $\mathrm{Y}$ (王毅), Xu F-S(徐芳森). Construction of two DH populations and identification of chromosome ploidy in burley tobacco. Acta Tab Sin (中国烟草学报), 2007, 13(2): 33-37 (in Chinese with English abstract)

[20] Li J(李佳), Shen B-Z(沈斌章), Han J-X(韩继祥), Gan L(甘莉). An effective procedure for extracting total DNA in rape. $J$ Huazhong Agric Univ (华中农业大学学报), 1994, 13(5): 521-523 (in Chinese with English abstract)

[21] Lu G-Y(陆光远), Yang G-S(杨光圣), Fu T-D(傅廷栋). Molecular Markers for the Dominant Genic Male Sterility Gene and the Suppressor Gene in Brassica napus and Their Applications. PhD Dissertation of Huazhong Agricultural University, 2003. pp 38-40 (in Chinese with English abstract)

[22] Li G, Qurios C F. Sequence-related amplified polymorphism (SRAP), a new marker system based on a simple PCR reaction:
Its application to mapping and gene tagging in Brassica. Theor Appel Genet, 2001, 103: 455-461

[23] Lincoln S, Daly M, Lander E S. Construction Genetic Maps with Mapmaker/Exp 3.0. Whitehead Institution Technical Report. 2nd edn. Cambridge, MA: Whitehead Institution, 1992. pp 1-49

[24] Liu R-H(刘仁虎), Meng J-L(孟金陵). MapDraw: A microsoft excel macro for drawing genetic linkage maps based on given genetic linkage data. Hereditas (Beijing) (遗传), 2003, 25(3): 317-321 (in Chinese with English abstract)

[25] Wang Z-D(王志德), Wang Y-Y(王元英), Mou J-M(牟建民), Dai P-G(戴培刚), Liu Y-H(刘艳华), Qian Y-M(钱玉梅), Kong F-Y(孔凡玉), Zhang H-B(张怀宝). Descriptors and Data Standard for Tobacco (Nicotiana spp.)(烟草种质资源描述规范和数 据标准). Beijing: China Agriculture Press, 2006. pp 12-36 (in Chinese)

[26] Determination of total plant alkaloid of tobacco and tobacco products-continuous flow method. Industry Standard of People Republic China (中华人民共和国行业标准), YC/T160-2002, Beijing: Standards Press of China, 2002. pp 1-6 (in Chinese)

[27] Zeng Z B. Precision mapping of quantitative trait loci. Genetics, 1993, 136: 1457-1468

[28] Cai C C, Tu J X, Fu T D, Chen B Y. The genetic basis of flowering time and photoperiod sensitivity in rapeseed Brassica napus L. Russian J Genet, 2008, 44: 326-333

[29] Edwards M D, Helentjaris T, Wright S. Molecular-marker- facilitated investigation if quantitative trait loci in maize: 4. Analysis based on genome saturation with isozyme and restriction fragment length polymorphism markers. Theor Appl Genet, 1992, 83: $765-774$

\section{欢迎订阅 2010 年《分子植物育种》}

《分子植物育种》是一份为转基因育种、分子标记辅助育种及常规育种服务的科学杂志, 也是中国唯一 的一份以育种为名的科学杂志。创刊于 2003 年, 创刊伊始即被美国化学文摘(CA), 中国科学引文数据库、 中国科技期刊全文数据库、中国引文数据库、中国科技期刊数据库、中文科技期刊数据库、中国核心期刊(遴 选)数据库、中国生物学文摘和中国生物学数据库等多家国内外数据库和检索系统收录。据 2008 年《中国学 术期刊综合引证报告》登载, 本刊的影响因子为 1.230 。本刊设专题评述、研究论文、研究报告、专题介绍、 学位论文简报、新基因新种质新品种、新思路新技术新方法和信息索引等栏目, 主要刊登水稻、小麦、玉米、 油菜、大豆、棉麻、薯类、果树、蔬菜、花卉、茶叶、林草等植物的育种及相关领域研究成果，是了解中国 分子植物育种的一个重要窗口。本刊已建立了全英文的期刊网站, 定期发布学术动态、出版信息及期刊近期 目录等, 实现作者编者读者同步分享。

欢迎订阅 《分子植物育种》, 本刊单月 28 日出版, 国内定价: 40.00 元/期, 240.00 元/年; 国外定价: 20.00 美元/期, 120.00 美元/年。国内统一刊号: CN 46-1068/S, 国际标准刊号:ISSN 1672-416X, 邮发代号: 84-23。 读者可到当地邮局订阅, 或直接汇款至编辑部, 免收邮费。

地址: 海南省海口市海秀大道 128 号双岛公寓 13B 室(邮编: 570206)

电话: 0898-68966415; 传真: 0898-68958180

E-mail: mpb@hibio.org, mpb@molplantbreed.org; 网址: http://www.molplantbreed.org 